\title{
On Solution of Boundary Value Problems via Weak Contractions
}

\author{
Gopi Prasad $\mathbb{D}^{1}$ and Hüseyin Ișik $\mathbb{D}^{2}$ \\ ${ }^{1}$ Department of Mathematics, H.N.B. Garhwal University, 246174, Srinagar Garhwal, India \\ ${ }^{2}$ Department of Engineering Science, Bandırma Onyedi Eylül University, 10200 Bandırma, Turkey \\ Correspondence should be addressed to Hüseyin Işik; isikhuseyin76@gmail.com
}

Received 9 August 2021; Revised 9 November 2021; Accepted 1 December 2021; Published 11 January 2022

Academic Editor: Humberto Rafeiro

Copyright (C) 2022 Gopi Prasad and Hüseyin Ișik. This is an open access article distributed under the Creative Commons Attribution License, which permits unrestricted use, distribution, and reproduction in any medium, provided the original work is properly cited.

\begin{abstract}
The aim is to present a new relational variant of fixed point result that generalizes various fixed point results of the existing theme for contractive type mappings. As an application, we solve a periodic boundary value problem and validate all assertions with the help of nontrivial examples. We also highlight the close connections of the fixed point results equipped with a binary relation to that of graph related metrical fixed point results. Radically, these investigations unify the theory of metrical fixed points for contractive type mappings.
\end{abstract}

\section{Introduction}

Alber and Guerre-Delabriere [1] presented the notion of weak contraction in Hilbert spaces and established the compatible fixed point results. Afterwards, Rhoades [2] stated that these results are still valid in the settings of metric spaces which are complete. Weak contractions are also connected to the mappings of Boyd and Wong [3], Geraghty [4], and that of Reich [5]. Further, generalizations of these fixed point results for weakly contractive mappings on this theme was obtained by Dutta and Choudhury [6]. In this continuation, an ordered analog of results due to Reich [5] and Geraghty [4] were presented by Amini-Harandi and Emami [7]. However, an analog of Banach contraction principle [8] in the same settings was investigated by Turinici [9] which was later explored by several authors (see Ran and Reurings [10], Nieto and Rodríguez-López [11], Sabetghadam and Masiha [12], Sabetghadam et al. [13], Harjani and Sadarangani [14], Samet and Turinici [15], Alam and Imdad [16, 17], and Prasad $[18,19])$ and this process is still on. Meanwhile, Jachymski [20] presented an interesting metrical fixed point result by incorporating the notion of graphical contraction mapping, and there exist detailed generalization of this settings too (see for instance [21-23]).

Among all these generalizations, we must recite Alam and Imdad [17] in which the authors utilized relational var- iants of metrical definitions of continuity, contractions, and completeness to obtain some interesting generalizations of the fixed point results. Noticeably, Alam and Imdad [16] presented a relational variant of fixed point result due to Boyd and Wong [3] to such settings. The objective of this work is to investigate a new fixed point theorem in relational metric spaces and to solve a boundary value problem in the light of obtained results. Moreover, we highlight the connection of such findings to the fixed point results obtained under graphical contraction mappings. In this way, we utilize the contractive assumption enjoying only on those elements which are associated with either a binary relation or some graph related structure instead of the entire space.

\section{Preliminaries}

We use notations $\Re$ for a nonempty binary relation, $\mathbb{N}_{0}$ for the the set $\mathbb{N}_{0}=\mathbb{N} \cup\{0\}$, and $\mathbb{R}$ for the set of real numbers thoroughly in this paper. Also, the triple $(F, \varsigma, \Re)$ denotes an $\mathfrak{R}$-metric space where $\mathfrak{R}$ is a binary relation on a nonempty set $F$, and $\zeta$ is a metric on $F$.

Definition 1 (see [24]). Let $\mathrm{F}$ be a nonempty set and $\mathfrak{R} \subseteq \mathrm{F}$ $\times F$. Then, 
(a) $\mathfrak{R}$ is a binary relation on $\mathrm{F}$ and " $\breve{u}$ relates $\breve{v}$ under $\mathfrak{R}$ " iff $(\breve{u}, \breve{v}) \in \Re$

(b) $\breve{u}$ and $\breve{v}$ are $\Re$-comparative, if either $(\breve{u}, \breve{v}) \in \Re$ or ( $\breve{v}, \breve{u}) \in \mathfrak{R}$, and denoted by $[\breve{u}, \breve{v}] \in \Re$

(c) The inverse of $\mathfrak{R}$ is defined by $\mathfrak{R}^{-1}:=\left\{(\breve{u}, \breve{v}) \in \mathrm{F}^{2}\right.$ $:(\breve{v}, \breve{u}) \in \Re\}$

(d) The symmetric closure of $\mathfrak{R}$ is defined by $\mathfrak{R}^{s}:=\mathfrak{R}$ $\cup \mathfrak{R}^{-1}$

(e) $(\breve{u}, \breve{v}) \in \Re^{s}$ iff $[\breve{u}, \breve{v}] \in \mathfrak{R}$

Definition 2 (see [17]). Consider a binary relation $\mathfrak{R}$ and a self-map $\theta$ on a nonempty set $\mathrm{F}$. Then, for $\breve{u}, \breve{v} \in \mathrm{F}$,

(a) $\mathfrak{R}$ is $\theta$-closed if

$$
(\breve{u}, \breve{v}) \in \mathfrak{R} \Rightarrow(\theta \breve{u}, \theta \breve{v}) \in \mathfrak{R}, \breve{u}, \breve{v} \in \mathrm{F} .
$$

(b) $\mathfrak{R}$ is $\theta$-closed iff $\mathfrak{R}^{s}$ is $\theta$-closed

Definition 3 (see [17]). Consider a binary relation $\mathfrak{R}$ and a sequence $\left\{\breve{u}_{n}\right\}$ on a nonempty set $\mathrm{F}$. Then, $\left\{\breve{u}_{n}\right\}$ is an $\mathfrak{R}$ -preserving sequence (shortly, $\mathfrak{R}$-sequence) if $\left(\breve{u}_{n}, \breve{u}_{n+1}\right) \in$ $\mathfrak{R}, n \in \mathbb{N}_{0}$.

Definition 4 (see [17]). Consider an $\Re$-sequence $\left\{\breve{u}_{n}\right\}$ on an $\mathfrak{R}$-metric space $(\mathrm{F}, \varsigma, \mathfrak{R})$. Then, $(\mathrm{F}, \varsigma, \mathfrak{R})$ is $\mathfrak{R}$-complete if every $\mathfrak{R}$-Cauchy sequence converges to a point in $F$.

Remark 5. Every $\mathfrak{R}$-complete metric space is a complete metric space, and in respect to the universal relation, these notions are the same.

Proposition 6 ([17]). Consider a binary relation $\mathfrak{R}$ and $a$ self-map $\theta$ on a nonempty set $\mathrm{F}$. If $\mathfrak{R}$ is $\theta$-closed, then $\mathfrak{R}$ is $\theta^{n}$-closed, where $n \in \mathbb{N}_{0}$ and $\theta^{n}$ denotes $n$th iterate of $\theta$.

Definition 7 (see [17]). Consider a self-map $\theta$ on an $\mathfrak{R}$ -metric space $(F, \varsigma, \mathfrak{R})$. Then, $\theta$ is $\mathfrak{R}$-continuous at $\breve{u}$ if for any $\Re$-sequence $\left\{\breve{u}_{n}\right\}$ with $\breve{u}_{n} \longrightarrow^{\varsigma} \breve{u}$, we have $\theta \breve{u}_{n} \longrightarrow^{\varsigma} \theta \breve{u}$. Moreover, $\theta$ is $\mathfrak{R}$-continuous if it is $\mathfrak{R}$-continuous at each point of $F$.

Remark 8 . Noticeably, continuity of $\theta$ implies $\mathfrak{R}$-continuity, and in respect to the universal relation, these notions are the same.

Definition 9 (see [17]). Consider an $\mathfrak{R}$-metric space $(F, \varsigma, \mathfrak{R})$ . Then, $\mathfrak{R}$ is $\varsigma$-self-closed if for any $\mathfrak{R}$-sequence $\left\{\breve{u}_{n}\right\} \subset \mathrm{F}$ with $\breve{u}_{n} \longrightarrow \varsigma \breve{u}$, there exists a subsequence $\left\{\breve{u}_{n_{k}}\right\}$ of $\left\{\breve{u}_{n}\right\}$ with $\left[\breve{u}_{n_{k}}, \breve{u}\right] \in \Re, k \in \mathbb{N}_{0}$.
Definition 10 (see [17]). Consider a binary relation $\mathfrak{R}$ on a nonempty set F. A subset $\mathscr{E}$ of $F$ is $\Re$-connected if for each pair $\breve{u}, \breve{v} \in \mathscr{E}$; there exists a path in $\Re$ from $\breve{u}$ to $\breve{v}$.

Definition 11 (see [25]). Consider a binary relation $\mathfrak{R}$ on a nonempty set $F$. Then, a subset $\mathscr{E}$ of $F$ is $\mathfrak{R}$-directed if for each pair $\breve{u}, \breve{v} \in \mathscr{E}$; there exists $\breve{w} \in F$ so that $(\breve{u}, \breve{w}) \in \mathfrak{R}$ and $(\breve{v}, \breve{w}) \in \mathfrak{R}$.

Definition 12 ([16]). Consider a self-map $\theta$ on an $\mathfrak{R}$-metric space $(\mathrm{F}, \varsigma, \mathfrak{R})$. Then, $\mathfrak{R}$ is $\theta$-transitive if for any $\breve{u}, \breve{v}, \breve{w} \in \mathrm{F}$ $,(\theta \breve{u}, \theta \breve{v}),(\theta \breve{v}, \theta \breve{w}) \in \mathfrak{R} \Rightarrow(\theta \breve{u}, \theta \breve{w}) \in \mathfrak{R}$.

Motivated by Turinici [26], Alam and Imdad [16] notified the subsequent weaker form of transitivity.

Definition 13 ([16]). A binary relation $\mathfrak{R}$ on a nonempty set $F$ is locally transitive if for each (effectively) $\mathfrak{R}$-sequence \{ $\left.\breve{u}_{n}\right\} \subset \mathrm{F}$ (with range $\mathcal{U}:=\left\{\breve{u}_{n}: n \in N_{0}\right\}$ ), the binary relation $\left.\mathfrak{R}\right|_{\mathscr{U}}$ is transitive, where $\left.\mathfrak{R}\right|_{\mathscr{U}}$ is the restriction of $\mathfrak{R}$ to $\mathcal{U}$.

Definition 14 ([16]). Consider a self-map $\theta$ on an $\mathfrak{R}$-metric space $(F, \varsigma, \mathfrak{R})$. Then, $\mathfrak{R}$ is locally $\theta$-transitive if for each (effectively) $\mathfrak{R}$-sequence $\left\{\breve{u}_{n}\right\} \subset \theta(\mathrm{F})$ (with range $\mathcal{U}:=\left\{\breve{u}_{n}\right.$ $\left.: n \in N_{0}\right\}$ ), the binary relation $\left.\mathfrak{R}\right|_{\mathscr{U}}$ is transitive.

Definition 15 (see [25]). Consider a binary relation $\mathfrak{R}$ on a nonempty set $\mathrm{F}$. For $\breve{u}, \breve{v} \in \mathrm{F}$, a path of length $k(k \in \mathbb{N})$, in $\Re$ from $\breve{u}$ to $\breve{v}$ is a finite sequence $\left\{\breve{w}_{0}, \breve{w}_{1}, \breve{w}_{2}, \cdots, \breve{w}_{k}\right\} \subset \mathrm{F}$ satisfying the following:

(i) $\breve{w}_{0}=\breve{u}$ and $\breve{w}_{k}=\breve{v}$

(ii) $\left(\breve{w}_{i}, \breve{w}_{i+1}\right) \in \Re$ for each $i(0 \leq i \leq k-1)$.

Noticeably, a path of length $k$ has $k+1$ elements of $\mathrm{F}$, though they are not necessarily distinct.

Lemma 16 (see $[16,27])$. Consider a sequence $\left\{\breve{u}_{n}\right\}$ on a metric space $(\mathrm{F}, \varsigma)$. If $\left\{\breve{u}_{n}\right\}$ is not a Cauchy, then there exist $\varepsilon>0$ and two subsequences $\left\{\breve{u}_{n_{k}}\right\}$ and $\left\{\breve{u}_{m_{k}}\right\}$ of $\left\{\breve{u}_{n}\right\}$ so that for $k \in \mathbb{N}$,

$$
\begin{gathered}
k \leq m_{k}<n_{k}, \\
\varsigma\left(\breve{u}_{m_{k}}, \breve{u}_{n_{k}}\right)>\varepsilon, \\
\varsigma\left(\breve{u}_{m_{k}}, \breve{u}_{n_{k}-1}\right) \leq \varepsilon .
\end{gathered}
$$

(i) Moreover, if $\lim _{n \longrightarrow \infty} \varsigma\left(\breve{u}_{n}, \breve{u}_{n+1}\right)=0$, then

$$
\begin{gathered}
\lim _{k \longrightarrow \infty} \varsigma\left(\breve{u}_{m_{k}}, \breve{u}_{n_{k}}\right)=\varepsilon, \\
\lim _{k \longrightarrow \infty} \varsigma\left(\breve{u}_{m_{k}+1}, \breve{u}_{n_{k}+1}\right)=\varepsilon .
\end{gathered}
$$

Consider a binary relation $\mathfrak{R}$ and a self-map $\theta$ on a nonempty set $\mathrm{F}$. We use the following notations in the subsequent sections: 
(i) $\mathscr{F}(\theta):=\{\breve{u} \in F: \breve{u}=\theta \breve{u}\}$ (the set of all fixed points of $\theta)$,

(ii) $\mathscr{M}(\theta, \mathfrak{R}):=\{\breve{u} \in \mathrm{F}:(\breve{u}, \theta \breve{u}) \in \mathfrak{R}\}$.

Also, $\mathscr{C}$ is the class of functions $\varphi:[0,+\infty) \longrightarrow[0,1)$ satisfying the assumption $\varphi\left(s_{n}\right) \longrightarrow 1$ implies $s_{n} \longrightarrow 0$.

\section{Main Results}

In this section, we first consider the existence and uniqueness of fixed points for contractive mappings in relational metric spaces. Secondly, we present results related to graphical structure in the similar metric settings.

Theorem 17. Consider a self-map $\theta$ on an $\mathfrak{R}$-metric space ( $\mathrm{F}, \varsigma, \mathfrak{R})$. Assume that the subsequent assumptions hold:

(a) $(\mathrm{F}, \varsigma, \mathfrak{R})$ is $\mathfrak{R}$-complete

(b) $\mathfrak{R}$ is $\theta$-closed and locally $\theta$-transitive

(c) either $\theta$ is $\mathfrak{R}$-continuous or $\mathfrak{R}$ is $\varsigma$-self-closed

(d) $\mathscr{M}(\theta, \mathfrak{R})$ is nonempty

(e) there exists $\varphi \in \mathscr{C}$ so that

$$
\varsigma(\theta \breve{u}, \theta \breve{v}) \leq \varphi(\varsigma(\breve{u}, \breve{v})) \varsigma(\breve{u}, \breve{v})
$$

for each $\breve{u}, \breve{v} \in \mathrm{F}$ with $(\breve{u}, \breve{v}) \in \mathfrak{R}$. Then, $\theta$ has a fixed point.

Proof. In the light of assumption $(d)$, let $\breve{u}_{0} \in \mathscr{M}(\theta, \mathfrak{R})$. Define a sequence $\left\{\breve{u}_{n}\right\}$ of joint iterates with initial point $\breve{u}_{0}$, that is,

$$
\breve{u}_{n}=\theta^{n} \breve{u}_{0}, n \in \mathbb{N}_{0}
$$

Since $\left(\breve{u}_{0}, \theta \breve{u}_{0}\right) \in \mathfrak{R}$ and $\mathfrak{R}$ is $\theta$-closed, we have

$$
\left(\theta \breve{u}_{0}, \theta^{2} \breve{u}_{0}\right),\left(\theta^{2} \breve{u}_{0}, \theta^{3} \breve{u}_{0}\right), \cdots,\left(\theta^{n} \breve{u}_{0}, \theta^{n+1} \breve{u}_{0}\right), \cdots \in \mathfrak{R},
$$

so that

$$
\left(\breve{u}_{n}, \breve{u}_{n+1}\right) \in \Re, n \in \mathbb{N}_{0} \text {. }
$$

So, $\left\{\breve{u}_{n}\right\}$ is $\mathfrak{R}$-sequence.

If there exists $n_{0} \in \mathbb{N}$ so that $\varsigma\left(\breve{u}_{n_{0}}, \breve{u}_{n_{0}-1}\right)=0$, then $\breve{u}_{n_{0}}$ $=\theta \breve{u}_{n_{0}-1}=\breve{u}_{n_{0}-1}$ is a fixed point of $\theta$, so the proof is accomplished.

In the other case, assume that $\varsigma\left(\breve{u}_{n}, \breve{u}_{n-1}\right) \neq 0, n \in \mathbb{N}$. From (e), we have

$\varsigma\left(\breve{u}_{n+1}, \breve{u}_{n}\right)=\varsigma\left(\theta \breve{u}_{n}, \theta \breve{u}_{n-1}\right) \leq \varphi\left(\varsigma\left(\breve{u}_{n}, \breve{u}_{n-1}\right)\right) \varsigma\left(\breve{u}_{n}, \breve{u}_{n-1}\right)<\varsigma\left(\breve{u}_{n}, \breve{u}_{n-1}\right)$.

Put $s_{n}:=\varsigma\left(\breve{u}_{n+1}, \breve{u}_{n}\right)$. Then, we have

$$
s_{n} \leq \varphi\left(s_{n-1}\right) s_{n-1}<s_{n-1} .
$$

So, $\left\{s_{n}\right\}$ is a nonnegative nonincreasing and bounded below which possesses the limit $s$. From the inequality (9), taking $n \longrightarrow \infty$, we have

$$
s \leq \varphi(s) s<s
$$

implies $\varphi(s)=1$, and so, $s=0$.

Now, we shall show that $\left\{\breve{u}_{n}\right\}$ is Cauchy. On contrary, assume that $\left\{\breve{u}_{n}\right\}$ is not Cauchy. So, by Lemma 16 , there exist $\varepsilon>0$ and two subsequences $\left\{\breve{u}_{n_{k}}\right\}$ and $\left\{\breve{u}_{m_{k}}\right\}$ of $\left\{\breve{u}_{n}\right\}$ so that

$$
k \leq m_{k} \leq n_{k}, \varsigma\left(\breve{u}_{m_{k}}, \breve{u}_{n_{k}}\right)>\varepsilon \geq \varsigma\left(\breve{u}_{m_{k}}, \breve{u}_{n_{k}-1}\right), k \in \mathbb{N} .
$$

Next, in view of Lemma 16, we have

$$
\lim _{k \longrightarrow \infty} \varsigma\left(\breve{u}_{m_{k}}, \breve{u}_{n_{k}}\right)=\lim _{k \longrightarrow \infty} \varsigma\left(\breve{u}_{m_{k}+1}, \breve{u}_{n_{k}+1}\right)=\varepsilon .
$$

Since $\left\{\breve{u}_{n}\right\}$ is $\mathfrak{R}$-sequence and $\left\{\breve{u}_{n}\right\} \subset \theta(\mathrm{F})$, so the local $\theta$ -transitivity of $\mathfrak{R}$ gives rise that $\left(\breve{u}_{m_{k}}, \breve{u}_{n_{k}}\right) \in \mathfrak{R}$. By triangular inequality and $(e)$, we obtain

$$
\begin{aligned}
\varsigma\left(\breve{u}_{m_{k}}, \breve{u}_{n_{k}}\right) & \leq \varsigma\left(\breve{u}_{m_{k}}, \breve{u}_{m_{k}+1}\right)+\varsigma\left(\breve{u}_{m_{k}+1}, \breve{u}_{n_{k}+1}\right)+\varsigma\left(\breve{u}_{n_{k}}, \breve{u}_{n_{k}+1}\right) \\
& \leq \varsigma\left(\breve{u}_{m_{k}}, \breve{u}_{m_{k}+1}\right)+\varphi\left(\varsigma\left(\breve{u}_{m_{k}}, \breve{u}_{n_{k}}\right)\right) \varsigma\left(\breve{u}_{m_{k}}, \breve{u}_{n_{k}}\right)+\varsigma\left(\breve{u}_{n_{k}}, \breve{u}_{n_{k}+1}\right)
\end{aligned}
$$

that is,

$$
\varsigma\left(\breve{u}_{m_{k}}, \breve{u}_{n_{k}}\right) \leq\left(1-\varphi\left(\varsigma\left(\breve{u}_{m_{k}}, \breve{u}_{n_{k}}\right)\right)\right)^{-1}\left[\varsigma\left(\breve{u}_{m_{k}}, \breve{u}_{m_{k}+1}\right)+\varsigma\left(\breve{u}_{n_{k}}, \breve{u}_{n_{k}+1}\right)\right] .
$$

Using the facts that $\limsup _{k \longrightarrow+\infty} \varsigma\left(\breve{u}_{m_{k}}, \breve{u}_{n_{k}}\right)>0$ and $\lim _{n \longrightarrow+\infty} \varsigma\left(\breve{u}_{n_{k}}, \breve{u}_{n_{k}+1}\right)=0$, we have

$$
\limsup _{k \longrightarrow+\infty}\left(1-\varphi\left(\varsigma\left(\breve{u}_{m_{k}}, \breve{u}_{n_{k}}\right)\right)\right)^{-1}=+\infty
$$

which implies that $\limsup _{k \longrightarrow+\infty} \varphi\left(\varsigma\left(\breve{u}_{m_{k}}, \breve{u}_{n_{k}}\right)\right)=1$. Since $\varphi$ $\in \mathscr{C}$, we obtain

$$
\underset{k \longrightarrow+\infty}{\limsup }\left(\breve{u}_{m_{k}}, \breve{u}_{n_{k}}\right)=0
$$

which is a contradiction in the light of (12). So, $\left\{\breve{u}_{n}\right\}$ is $\mathfrak{R}$ Cauchy. As $(F, \varsigma, \mathfrak{R})$ is $\mathfrak{R}$-complete, there exists $\breve{u} \in F$ so that $\breve{u}_{n} \longrightarrow{ }^{\varsigma} \breve{u}$.

Next, we assert that $\breve{u}$ is a fixed point of $\theta$. At first, we consider $\theta$ is $\mathfrak{R}$-continuous. As $\left\{\breve{u}_{n}\right\}$ is $\mathfrak{R}$-sequence with $\breve{u}_{n} \longrightarrow \varsigma \breve{u}$, $\mathfrak{R}$-continuity of $\theta$ implies that $\breve{u}_{n+1}=\theta \breve{u}_{n} \longrightarrow^{\varsigma} \theta$ $\breve{u}$. From the uniqueness of the limit, we obtain $\theta \breve{u}=\breve{u}$, that is, $\breve{u}$ is a fixed point of $\theta$.

Alternately, assume that $\mathfrak{R}$ is $\varsigma$-self-closed. So, there exists subsequence $\left\{\breve{u}_{n_{k}}\right\}$ of $\left\{\breve{u}_{n}\right\}$ with $\left[\breve{u}_{n_{k}}, \breve{u}\right] \in \Re, k \in \mathbb{N}_{0}$. 
By using the fact that $\left[\breve{u}_{n_{k}}, \breve{u}\right] \in \Re$ in the light of $(e)$, we have

$$
\varsigma\left(\breve{u}_{n_{k}+1}, \theta \breve{u}\right)=\varsigma\left(\theta \breve{u}_{n_{k}}, \theta \breve{u}\right) \leq \varphi\left(\varsigma\left(\breve{u}_{n_{k}}, \breve{u}\right)\right) \varsigma\left(\breve{u}_{n_{k}}, \breve{u}\right)<\varsigma\left(\breve{u}_{n_{k}}, \breve{u}\right) .
$$

Taking limit $k \longrightarrow \infty$ and $\breve{u}_{n_{k}} \longrightarrow^{\varsigma} \breve{u}$, we have $\breve{u}_{n_{k}+1}$ $\longrightarrow^{\varsigma} \theta \breve{u}$, and hence, $\theta \breve{u}=\breve{u}$.

Remark 18. Theorem 17 remains valid if we consider $\theta$ -transitive, locally transitive or simply transitive assumption in place of the locally $\theta$-transitivity of $\Re$ besides retaining all other assumptions.

\subsection{Uniqueness Result}

Theorem 19. Along with the assumptions of Theorem 17, assume that the subsequent assumption holds: point.

(u) $\theta(\mathrm{F})$ is $\mathfrak{R}^{s}$-connected. Then, $\theta$ has a unique fixed

Proof. Let $\breve{u}$ and $\breve{v}$ be two distinct fixed points of $\theta$, that is, $\mathscr{F}(\theta) \neq \varnothing$ and $\breve{u}, \breve{v} \in \mathscr{F}(\theta)$, then for $n \in \mathbb{N}_{0}$, we have

$$
\theta^{n} \breve{u}=\breve{u}, \theta^{n} \breve{v}=\breve{v} .
$$

Noticeably, $\breve{u}, \breve{v} \in \theta(\mathrm{F})$. By assumption $(u)$, there exists a path (say $\breve{w}_{0}, \breve{w}_{1}, \breve{w}_{2}, \cdots, \breve{w}_{k}$ ) of finite length $k$ in $\Re^{s}$ from $\breve{u}$ to $\breve{v}$ so that

$$
\breve{w}_{0}=\breve{u}, \breve{w}_{k}=\breve{v} \text { and }\left[\breve{w}_{i}, \breve{w}_{i+1}\right] \in \Re \text { for each } i(0 \leq i \leq k-1) \text {. }
$$

As $\Re$ is $\theta$-closed, then in the light of Proposition 6, we obtain

$$
\left[\theta^{n} \breve{w}_{i}, \theta^{n} \breve{w}_{i+1}\right] \in \Re \text { for each } i(0 \leq i \leq k-1), n \in \mathbb{N}_{0} .
$$
obtain

Now, applying the contractive assumption (e) to (20), we

$\varsigma\left(\theta^{n} \breve{w}_{i}, \theta^{n} \breve{w}_{i+1}\right) \leq \varphi\left(\left(\varsigma\left(\theta^{n-1} \breve{w}_{i}, \theta^{n-1} \breve{w}_{i+1}\right)\right) \varsigma\left(\theta^{n-1} \breve{w}_{i}, \theta^{n-1} \breve{w}_{i+1}\right)\right.$

For convenience, we put $t_{n}^{i}=\varsigma\left(\theta^{n} \breve{w}_{i}, \theta^{n} \breve{w}_{i+1}\right)$.

We have two cases: Firstly, assume that $t_{n_{0}}^{i}=\varsigma\left(\theta^{n_{0}} \breve{w}_{i}\right.$, $\left.\theta^{n_{0}} \breve{w}_{i+1}\right)=0$ for some $n_{0} \in \mathbb{N}_{0}$, that is, $\theta^{n_{0}} \breve{w}_{i}=\theta^{n_{0}} \breve{w}_{i+1}$, which implies that $\theta^{n_{0}+1} \breve{w}_{i}=\theta^{n_{0}+1} \breve{w}_{i+1}$. In this way, $t_{n_{0}+1}^{i}=\varsigma\left(\theta^{n_{0}+1}\right.$ $\left.\breve{w}_{i}, \theta^{n_{0}+1} \breve{w}_{i+1}\right)=0$. Thus, by induction, we get $t_{n_{0}}^{i}=0$ for every $n \geq n_{0}$. Hence, $\lim _{n \rightarrow \infty} t_{n}^{i}=0$.

Secondly, assume that $t_{n}^{i}>0$ for $n \in \mathbb{N}_{0}$, then using (20), in view of $(e)$ and taking $n \longrightarrow \infty$ on the inequality (21), we have $\lim _{n \longrightarrow \infty} t_{n}^{i}=0$ for each $i(0 \leq i \leq k-1)$.
Finally, utilizing the triangular inequality of metric $\varsigma$, in view of above conclusion, we obtain

$$
\varsigma(\breve{u}, \breve{v})=\varsigma\left(\theta^{n} \breve{w}_{0}, \theta^{n} \breve{w}_{k}\right) \leq t_{n}^{0}+t_{n}^{1}+\cdots+t_{n}^{k-1} \longrightarrow 0,
$$

as $n \longrightarrow \infty$. Hence, $\theta$ has a unique fixed point.

Remark 20. Theorem 19 remains valid if we consider $\left.\mathfrak{R}\right|_{\theta(\mathrm{F})}$ is complete or $\theta(\mathrm{F})$ is $\mathfrak{R}^{s}$-directed in place of the assumption $(u)$ besides retaining the all other assumptions.

Example 21. Let $\mathrm{F}=[0,+\infty)$ equipped with the usual metric $\varsigma(\breve{u}, \breve{v})=|\breve{u}-\breve{v}|$ for $\breve{u}, \breve{v} \in \mathrm{F}$. Define a binary relation $\mathfrak{R}=\{($ $\breve{u}, \breve{v}): \breve{u} \geq \breve{v}$ and $\breve{u}, \breve{v} \in[0,1]\}$ on $\mathrm{F}$ and a mapping $\theta: \mathrm{F} \longrightarrow \mathrm{F}$ by

$$
\theta \breve{u}= \begin{cases}\frac{\breve{u}}{1+\breve{u}}, & \text { if } \breve{u} \in[0,1], \\ 3 \breve{u}, & \text { if } \breve{u} \in(1,+\infty) .\end{cases}
$$

Clearly, $(F, \varsigma, \Re)$ is an $\mathfrak{R}$-complete metric space and $\theta$ is $\Re$-continuous. Let $\breve{u}, \breve{v} \in \mathrm{F}$. If $(\breve{u}, \breve{v}) \in \Re$, that is, $\breve{u}, \breve{v} \in[0,1]$, then $(\theta \breve{u}, \theta \breve{v}) \in \Re$. Also, for all $\breve{u} \in[0,1]$, we have $\theta \breve{u} \leq 1$. This implies that $(\breve{u}, \theta \breve{u}) \in \Re$. Thus, the claim holds. In consequence of the above reasonings, $(0, \theta 0) \in \mathfrak{R}$. Also, we can easily verify that $\Re$ is $\theta$-transitive and locally $\theta$-transitive.

Let $\breve{u}, \breve{v} \in \mathrm{F}$ with $(\breve{u}, \breve{v}) \in \Re$. Define $\varphi(t)=(1 /(1+t)), t$ $\epsilon[0,+\infty)$, we have

$$
\begin{aligned}
\varsigma(\theta \breve{u}, \theta \breve{v})=|\theta \breve{u}-\theta \breve{v}| & =\left|\frac{\breve{u}}{1+\breve{u}}-\frac{\breve{v}}{1+\breve{v}}\right|=\frac{\breve{u}-\breve{v}}{1+\breve{u}+\breve{v}+\breve{u} \breve{v}} \\
& \leq \frac{\breve{u}-\breve{v}}{1+\breve{u}-\breve{v}}=\varphi(\varsigma(\breve{u}, \breve{v})) \varsigma(\breve{u}, \breve{v}) .
\end{aligned}
$$

Thus, all the assumptions of Theorems 17 and 19 are satisfied. Hence, $\theta$ has a unique fixed point.

Example 22. Let $\mathrm{F}=[1,4]$ equipped with the usual metric $\varsigma($ $\breve{u}, \breve{v})=|\breve{u}-\breve{v}|$. Define a binary relation $\Re=\{(1,1),(3 / 2,2)$, $(2,1),(2,2),(5 / 2,3),(3,3),(7 / 2,4)\}$ on $F$ and the mapping $\theta: \mathrm{F} \longrightarrow \mathrm{F}$ by

$$
\theta \breve{u}= \begin{cases}1, & \text { if } \breve{u} \in[1,2], \\ 2, & \text { if } \breve{u} \in(2,3], \\ 3, & \text { if } \breve{u} \in(3,4] .\end{cases}
$$

Let $(\breve{u}, \breve{v}) \in \Re$. Then, $(\theta \breve{u}, \theta \breve{v}) \in\{(1,1),(2,2),(3,3)\}$ which implies that $\mathfrak{R}$ is $\theta$-closed. Observe that $\mathfrak{R}$ is not reflexive, antisymmetric, and neither transitive. So, $\mathfrak{R}$ is not partial order.

Now, we shall show $R$ is $\varsigma$-self-closed. Let $\left\{\breve{u}_{n}\right\}$ be any $\Re$-sequence with $\breve{u}_{n} \longrightarrow^{\varsigma} \breve{u}$, so that $\left(\breve{u}_{n}, \breve{u}_{n+1}\right) \in \Re, n \in \mathbb{N}_{0}$ which implies that $\left\{\breve{u}_{n}\right\} \subset\{1,2,3\}$. As $\{1,2,3\}$ is closed, we can take a subsequence $\left\{\breve{u}_{n_{k}}\right\}$ of $\left\{\breve{u}_{n}\right\}$ so that $\breve{u}_{n_{k}}=\breve{u}, k$ 
$\in \mathbb{N}_{0}$, which implies that $\left[\breve{u}_{n_{k}}, \breve{u}\right] \in \Re, k \in \mathbb{N}_{0}$. Hence, $\mathfrak{R}$ is $\varsigma$ -self-closed.

Notice that, for $\varphi(t)=1 /(1+t), t \in[0,+\infty)$, we have

$$
\varsigma(\theta 1, \theta 3)=|\theta 1-\theta 3|=1>\varphi(\varsigma(1,3)) \varsigma(1,3)=\frac{2}{3},
$$

that is, $\theta$ does not satisfy the contractive assumption $(e)$ of Theorem 17. However, if $(\breve{u}, \breve{v}) \in \Re$, then the assumption (e) is satisfied for all $(\breve{u}, \breve{v}) \in \mathfrak{R}$. Also, by usual calculations, we can easily verify that $\theta(\mathrm{F})$ is $\mathfrak{R}^{s}$-connected.

So, $\theta$ satisfies all assumptions of Theorems 17 and 19. Thus, $\theta$ has a unique fixed point at $\breve{u}=1$.

Remark 23. Noticeably, in Example 22 binary relation $\mathfrak{R}$ is nonreflexive, nonsymmetric, nonantisymmetric, and nontransitive. So, it is not a partial order, quasiorder, and near-order which indicate the utility of such generalizations over the corresponding several prominent recent fixed point results on this theme.

3.2. Fixed Point Result under Graphical Structure. Jachymski [11] introduced the graphical variant of Banach contraction principle in metric spaces by transforming the order structure into a graphical structure on such spaces.

Let $\mathrm{F}$ be a nonempty set and $\Delta$ denotes the diagonal points of F $\times F$. Then, $\mathscr{G}$ is a directed graph with the vertex set $\mathscr{V}(\mathscr{G})$ which coincides with $\mathrm{F}$, and the edge set $\mathscr{E}(\mathscr{G})$ containing its edges with all loops, that is, $\mathscr{E}(\mathscr{G}) \supseteq \Delta$. Additionally, assuming that $\mathscr{G}$ has no parallel edges, so we can symbolize $\mathscr{G}$ as a pair $(\mathscr{V}(\mathscr{G}), \mathscr{E}(\mathscr{G}))$. Also, we assume $\mathscr{G}$ as a weighted graph by assigning to each edge the distance between its vertices. If $\breve{u}$ and $\breve{v}$ are any vertices of a graph $\mathscr{G}$, then a path in $\mathscr{G}$ from $\breve{u}$ to $\breve{v}$ of length $k(k \in \mathbb{N})$ is a sequence $\left\{\breve{u}_{i}\right\}_{i=0}^{k}$ of $k+1$ vertices so that $\breve{u}_{0}=\breve{u}, \breve{u}_{k}=\breve{v}$ and $\left(\breve{u}_{n-1}, \breve{u}_{n}\right) \in \mathscr{E}(\mathscr{G})$ for $i=1,2,3, \cdots, k$. Graph $\mathscr{G}$ is connected if there is a path between any two of its vertices, and $\mathscr{G}$ is weakly connected if $\tilde{\mathscr{G}}$ is connected (see for details [21-23]).

The triple $(\mathrm{F}, \varsigma, \mathscr{G})$ denotes a $\mathscr{G}$-metric space where $\mathscr{G}$ is a graph on a nonempty set $\mathrm{F}$ and $\varsigma$ is a metric on $\mathrm{F}$.

Definition 24 (see [20]). Consider a self-map $\theta$ on a $\mathscr{G}$-metric space $(F, \varsigma, \mathscr{G})$. Then, $\theta$ is said to be $\mathscr{G}$-contraction if there exists $k \in(0,1)$ such that

$$
\forall \breve{u}, \breve{v} \in \mathrm{F}, \quad(\breve{u}, \breve{v}) \in \mathscr{E}(\mathscr{G}) \Rightarrow \varsigma(\theta \breve{u}, \theta \breve{v}) \leq k \varsigma(\breve{u}, \breve{v}),
$$

and $\mathscr{G}$ is $\theta$-closed, that is,

$$
\forall \breve{u}, \breve{v} \in \mathrm{F}, \quad(\breve{u}, \breve{v}) \in \mathscr{E}(\mathscr{G}) \Rightarrow(\theta \breve{u}, \theta \breve{v}) \in \mathscr{E}(\mathscr{G}) .
$$

Definition 25 (see [20]). Consider a sequence $\left\{\breve{u}_{n}\right\}$ on a $\mathscr{G}$ -metric space $(F, \varsigma, \mathscr{G})$. Then, $\left\{\breve{u}_{n}\right\}$ is said to be edgepreserving sequence (shortly, $\mathscr{E}$-sequence) if $\left(\breve{u}_{n}, \breve{u}_{n+1}\right) \in \mathscr{E}$ $(\mathscr{G}), n \in \mathbb{N}_{0}$.

Also, $(\mathrm{F}, \varsigma, \mathscr{G})$ is $\mathscr{G}$-complete if every $\mathscr{E}$-Cauchy sequence converges in $\mathrm{F}$.
Definition 26 (see [20]). Consider a self-map $\theta$ on a $\mathscr{G}$-metric space $(F, \varsigma, \mathscr{G})$. Then, $\theta$ is $\mathscr{G}$-continuous at $\breve{u}$ if for any $\mathscr{E}$ -sequence $\left\{\breve{u}_{n}\right\}$ with $\breve{u}_{n} \longrightarrow^{\varsigma} \breve{u}$, we have $\theta \breve{u}_{n} \longrightarrow^{\varsigma} \theta \breve{u}$. Moreover, $\theta$ is $\mathscr{G}$-continuous if it is $\mathscr{G}$-continuous at each point of F.

Definition 27. Consider a $\mathscr{G}$-metric space $(\mathrm{F}, \varsigma, \mathscr{G})$. Then, $\mathscr{G}$ is $\varsigma$-self-closed if for any $\mathscr{E}$-sequence $\left\{\breve{u}_{n}\right\} \subset \mathrm{F}$ with $\breve{u}_{n} \longrightarrow \varsigma$ $\breve{u}$, there exists a subsequence $\left\{\breve{u}_{n_{k}}\right\}$ of $\left\{\breve{u}_{n}\right\}$ with $\left(\breve{u}_{n_{k}}, \breve{u}\right) \in$ $\mathscr{E}(\mathscr{G}), k \in \mathbb{N}_{0}$.

Definition 28 ([23]). Consider a graph $\mathscr{G}$ on a nonempty set F. Then, $\mathscr{G}$ is transitive if, for any $\breve{u}, \breve{v}, \breve{w} \in \mathscr{V}(\mathscr{G})$ with $(\breve{u}, \breve{v}$ ),$(\breve{v}, \breve{w}) \in \mathscr{E}(\mathscr{G}) \Rightarrow(\breve{u}, \breve{w}) \in \mathscr{E}(\mathscr{G})$.

Definition 29. Consider a self-map $\theta$ on a $\mathscr{G}$-metric space ( $\mathrm{F}, \varsigma, \mathscr{G})$. Then, $\mathscr{G}$ is $\theta$-transitive if for any $\breve{u}, \breve{v}, \breve{w} \in \mathrm{F},(\theta \breve{u}, \theta \breve{v}$ ),$(\theta \breve{v}, \theta \breve{w}) \in \mathscr{E}(\mathscr{G}) \Rightarrow(\theta \breve{u}, \theta \breve{w}) \in \mathscr{E}(\mathscr{G})$.

Definition 30. A graph $\mathscr{G}$ on a nonempty set F is locally transitive if for each (effectively) $\mathscr{E}$-sequence $\left\{\breve{u}_{n}\right\} \subset \mathrm{F}$ (with range $\left.\mathscr{U}:=\left\{\breve{u}_{n}: n \in \mathbb{N}_{0}\right\}\right)$, the graph $\left.\mathscr{G}\right|_{\mathscr{U}}$ is transitive.

Definition 31. Consider a self-map $\theta$ on a $\mathscr{G}$-metric space ( $\mathrm{F}, \varsigma, \mathscr{G})$. Then, $\mathscr{G}$ is locally $\theta$-transitive if for each (effectively) $\mathscr{E}$-sequence $\left\{\breve{u}_{n}\right\} \subset \theta(\mathrm{F})$ (with range $\mathcal{U}:=\left\{\breve{u}_{n}: n \in \mathbb{N}_{0}\right\}$ ), the graph $\left.\mathscr{G}\right|_{\mathscr{U}}$ is transitive.

Theorem 32. Consider a self-map $\theta$ on a $\mathscr{G}$-metric space (F $, \varsigma, \mathscr{G})$. Assume that the subsequent assumptions hold:

(a) $(\mathrm{F}, \varsigma, \mathscr{G})$ is $\mathscr{G}$-complete

(b) $\mathscr{G}$ is $\theta$-closed and locally $\theta$-transitive

(c) either $\theta$ is $\mathscr{G}$-continuous or $\mathscr{G}$ is $\varsigma$-self-closed

(d) $\mathscr{M}(\theta, \mathscr{G})$ is nonempty, that is, there exists $\breve{u}_{0}$ in $\mathrm{F}$ so that $\left(\breve{u}_{0}, \theta \breve{u}_{0}\right) \in \mathscr{E}(\mathscr{G})$,

(e) there exists $\varphi \in \mathscr{C}$ so that

$$
\varsigma(\theta \breve{u}, \theta \breve{v}) \leq \varphi(\varsigma(\breve{u}, \breve{v})) \varsigma(\breve{u}, \breve{v})
$$

for all $\breve{u}, \breve{v} \in \mathrm{F}$ with $(\breve{u}, \breve{v}) \in \mathscr{E}(\mathscr{G})$,

(f) $\mathscr{G}$ is weakly connected

Then, $\theta$ has a unique fixed point.

Proof. Define $\mathfrak{R}=\{(\breve{u}, \breve{v}):(\breve{u}, \breve{v}) \in \mathscr{E}(\mathscr{G}), \breve{u}, \breve{v} \in F\}$. Then, clearly, the contractive assumption $(e)$ is same as in Theorem 17. Similarly, $\mathscr{G}$-completeness of metric space implies the $\mathfrak{R}$-completeness. From $(d)$, we have $\left(\breve{u}_{0}, \theta \breve{u}_{0}\right) \in \mathscr{E}(\mathscr{G})$, which implies that $\mathscr{M}(\theta, \Re)$ is nonempty. For $\breve{u}, \breve{v} \in \mathrm{F}$ with $(\breve{u}, \breve{v}) \in \mathscr{E}(\mathscr{G}) \Rightarrow(\breve{u}, \breve{v}) \in \Re$, then in the light of assumption (b), $(\theta \breve{u}, \theta \breve{v}) \in \mathscr{E}(\mathscr{G}) \Rightarrow(\theta \breve{u}, \theta \breve{v}) \in \mathfrak{R}$, that is, if $\mathscr{G}$ is $\theta$-closed and locally $\theta$-transitive, then $\mathfrak{R}$ is $\theta$-closed and locally $\theta$ -transitive. Also, one can easily verify that $\mathscr{G}$-continuity of $\theta$ implies $\mathfrak{R}$-continuity and $\varsigma$-self-closedness of $\mathscr{G}$ implies $\varsigma$ 
-self-closedness of $\mathfrak{R}$. Moreover, the assumption $(f)$ implies that $\theta(\mathrm{F})$ is $\mathfrak{R}^{s}$-connected which validates that $\theta$ has only one fixed point.

Remark 33. In view of the above discussion, if we define a binary relation $\mathfrak{R}$ so that $\mathfrak{R}=\{(\breve{u}, \breve{v}):(\breve{u}, \breve{v}) \in \mathscr{E}(\mathscr{G}), \breve{u}, \breve{v} \in$ $F\}$. Then, under this assumption of $\mathfrak{R}$, Theorem 32 reduces to Theorems 17 and 19. This implies that edge preserving structure of a graph is considered as a particular case of a binary relation $\mathfrak{R}$.

Remark 34. Noticeably, if we define $\mathscr{E}(\mathscr{G})$ so that $\mathscr{E}(\mathscr{G})=\{$ $(\breve{u}, \breve{v}): \breve{u} \leq \breve{v}, \breve{u}, \breve{v} \in F\}$. Then, under this assumption of $\mathscr{E}(\mathscr{G}$ ), Theorem 32 reduces to their corresponding partial ordered analogous. This implies that partial-order relationrelated metrical notions can be considered as a particular case of an edge-preserving structure related to a graph.

\section{An Application}

The theory of boundary value problems is a substantial field of mathematics, having various applications in numerous branches of physics, biology, chemistry, engineering, and other fields related to the real life problems. Based on this fact, we present a unique solution for the first order periodic boundary value problem by utilizing the main result. For this, we consider a periodic boundary value problem of first order as follows:

$$
\breve{u}^{\prime}(s)=q(s, \breve{u}(s)), s \in J=[0, S], \breve{u}(0)=\breve{u}(S),
$$

where $S>0$ and $q: J \times \mathbb{R} \longrightarrow \mathbb{R}$ is a continuous function.

Let $C(J)$ denote the space of all continuous functions defined on $J$. We recall the subsequent definitions.

Definition 35 (see [14]). A function $\beta \in C^{1}(J)$ is a lower solution of (30), if

$$
\begin{gathered}
\beta^{\prime}(s) \leq q(s, \beta(s)), s \in J, \\
\beta(0) \leq \beta(S) .
\end{gathered}
$$

Definition 36 ([14]). A function $\beta \in C^{1}(J)$ is a upper solution of (30), if

$$
\begin{gathered}
\beta^{\prime}(s) \geq q(s, \beta(s)), s \in J, \\
\beta(0) \geq \beta(S) .
\end{gathered}
$$

Now, we prove the existence of solution for the problem (30). Let $\mathscr{A}$ be a class of functions $\phi:[0,+\infty) \longrightarrow[0,+\infty)$ satisfying the subsequent assumptions:

(i) $\phi$ is increasing

(ii) for each $\breve{u}>0, \phi(\breve{u})<\breve{u}$

Examples of such functions are $\phi(s)=\mu s, s \in[0,1), \phi(s)$ $=s /(1+s)$ and $\phi(s)=\ln (1+s)$.
Theorem 37. In addition to the problem (30), assume that there exists $\delta>0$ so that $\breve{u}, \breve{v} \in \mathbb{R}$ with $\breve{u} \leq \breve{v}$,

$$
0 \leq q(s, \breve{v})+\delta \breve{v}-[q(s, \breve{u})+\delta \breve{u}] \leq \delta \phi(\breve{v}-\breve{u})
$$

where $\phi \in \mathscr{A}$. Then, the existence of a lower or an upper solution of problem (30) validates the existence and uniqueness of a solution of problem (30).

Proof. Problem (30) can be rewritten as

$$
\breve{u}^{\prime}(s)+\delta \breve{u}(s)=q(s, \breve{u}(s))+\delta \breve{u}(s), s \in J=[0, S], \breve{u}(0)=\breve{u}(S) .
$$

This can be transformed to the integral equation

$$
\breve{u}(s)=\int_{0}^{S} Q(s, r)[q(r, \breve{u}(r))+\delta \breve{u}(r)] d r,
$$

where

$$
Q(s, r)= \begin{cases}\frac{e^{\delta(S+r-s)}}{e^{\delta S}-1}, & 0 \leq r<s \leq S, \\ \frac{e^{\delta(r-s)}}{e^{\delta S}-1}, & 0 \leq s<r \leq S .\end{cases}
$$

Define $\theta: C(J) \longrightarrow C(J)$ by

$$
(\theta \breve{u})(s)=\int_{0}^{S} Q(s, r)[q(r, \breve{u}(r))+\delta \breve{u}(r)] d r,
$$

and a binary relation

$$
\mathfrak{R}=\{(\breve{u}, \breve{v}) \in C(J) \times C(J): \breve{u}(s) \leq \breve{v}(s), s \in J\}
$$

(i) Note that $C(J)$ with supmetric, that is, $\varsigma(\breve{u}, \breve{v})=\sup$ $|\breve{u}(s)-\breve{v}(s)|$ for $s \in J$ and $\breve{u}, \breve{v} \in C(J)$, is an $\Re$-complete metric space

(ii) For an $\mathfrak{R}$-preserving sequence $\left\{\breve{u}_{n}\right\}$ so that $\breve{u}_{n} \longrightarrow^{\varsigma}$ $\breve{w}$. Then, for $s \in J$, we have

$$
\breve{u}_{0}(s) \leq \breve{u}_{1}(s) \leq \breve{u}_{2}(s) \leq \cdots \leq \breve{u}_{n}(s) \leq \breve{u}_{n+1}(s) \leq \cdots
$$

which converges to $\breve{w}(s)$. This implies that $\breve{u}_{n}(s) \leq \breve{w}(s), n$ $\in \mathbb{N}_{0}$. So, $\left[\breve{u}_{n}, \breve{w}\right] \in \mathfrak{R}, n \in \mathbb{N}_{0}$. Hence, $\mathfrak{R}$ is $\varsigma$-self-closed.

(iii) For $(\breve{u}, \breve{v}) \in \Re$, that is, $\breve{u}(s) \leq \breve{v}(s)$, then in the light of inequality (33), we have

$$
q(s, \breve{u}(s))+\delta \breve{u}(s) \leq q(s, \breve{v}(s))+\delta \breve{v}(s), s \in J,
$$


and $Q(s, r)>0$ for $(s, r) \in J \times J$, we have

$$
\begin{aligned}
(\theta \breve{u})(s) & =\int_{0}^{S} Q(s, r)[q(r, \breve{u}(r))+\delta \breve{u}(r)] d r \\
& \leq \int_{0}^{S} Q(s, r)[q(r, \breve{v}(r))+\delta \breve{v}(r)] d r \\
& =(\theta \breve{v})(s), s \in J,
\end{aligned}
$$

so that $(\theta \breve{u}, \theta \breve{v}) \in \Re$, that is, $\Re$ is $\theta$-closed.

(iv) Let $\beta \in C^{1}(J)$ be a lower solution of (30), then we must have

$$
\beta^{\prime}(s)+\delta \beta(s) \leq q(s, \beta(s))+\delta \beta(s), s \in J .
$$

Multiplying both sides by $e^{\delta s}$, we have

$$
\left(\beta(s) e^{\delta s}\right)^{\prime} \leq[q(s, \beta(s))+\delta \beta(s)] e^{\delta s}, s \in J,
$$

so that

$$
\beta(s) e^{\delta s} \leq \beta(0)+\int_{0}^{s}[q(r, \beta(r))+\delta \beta(r)] e^{\delta r} d r, s \in J
$$

As $\beta(0) \leq \beta(S)$, we have

$$
\beta(0) e^{\delta S} \leq \beta(S) e^{\delta S} \leq \beta(0)+\int_{0}^{S}[q(r, \beta(r))+\delta \beta(r)] e^{\delta r} d r
$$

so that

$$
\beta(0) \leq \int_{0}^{S} \frac{e^{\delta r}}{e^{\delta S}-1}[q(r, \beta(r))+\delta \beta(r)] d r
$$

Using (43) and (45), we have

$$
\begin{aligned}
\beta(s) e^{\delta s} \leq & \int_{0}^{s} \frac{e^{\delta(S+r)}}{e^{\delta S}-1}[q(r, \beta(r))+\delta \beta(r)] d r \\
& +\int_{s}^{S} \frac{e^{\delta r}}{e^{\delta S}-1}[q(r, \beta(r))+\delta \beta(r)] d r,
\end{aligned}
$$

that is,

$$
\begin{aligned}
\beta(s) \leq & \int_{0}^{s} \frac{e^{\delta(S+r-s)}}{e^{\delta S}-1}[q(r, \beta(r))+\delta \beta(r)] d r \\
& +\int_{s}^{S} \frac{e^{\delta(r-s)}}{e^{\delta S}-1}[q(r, \beta(r))+\delta \beta(r)] d r \\
\leq & \int_{0}^{S} Q(s, r)[q(r, \beta(r))+\delta \beta(r)] d r=(\theta \beta)(s) .
\end{aligned}
$$

Thus, $(\beta(s), \theta \beta(s)) \in \mathfrak{R}, s \in J$ and so $\mathscr{M}(\theta, \mathfrak{R}) \neq \varnothing$.
For $(\breve{u}, \breve{v}) \in \Re$,

$$
\begin{aligned}
\varsigma(\theta \breve{u}, \theta \breve{v}) & =\sup _{s \in J}|(\theta \breve{u})(s)-(\theta \breve{v})(s)|=\sup _{s \in J}((\theta \breve{v})(s)-(\theta \breve{u})(s)) \\
& \leq \sup _{s \in J} \int_{0}^{S} Q(s, r)[q(r, \breve{v}(r))+\delta \breve{v}(r)-q(r, \breve{u}(r))-\delta \breve{u}(r)] d r \\
& \leq \sup _{s \in J} \int_{0}^{S} Q(s, r) \delta \phi(\breve{v}(r)-\breve{u}(r)) d r \leq \delta \phi(\varsigma(\breve{u}, \breve{v})) \sup _{s \in J} \int_{0}^{S} Q(s, r) d r \\
& =\delta \phi(\varsigma(\breve{u}, \breve{v})) \sup _{s \in J} \frac{1}{e^{\delta S}-1}\left(\left.\frac{1}{\delta} e^{\delta(S+r-s)}\right|_{0} ^{s}+\left.\frac{1}{\delta} e^{\delta(r-s)}\right|_{s} ^{S}\right) \\
& =\delta \phi(\varsigma(\breve{u}, \breve{v})) \frac{1}{\delta\left(e^{\delta S}-1\right)}\left(e^{\delta S}-1\right) \\
& =\phi(\varsigma(\breve{u}, \breve{v}))=\frac{\phi(\varsigma(\breve{u}, \breve{v}))}{\varsigma(\breve{u}, \breve{v})} \varsigma(\breve{u}, \breve{v}) .
\end{aligned}
$$

Define $\varphi(\breve{u})=\phi(\breve{u}) / \breve{u}$, then $\varphi \in \mathscr{C}$. By the last inequality, we derive

$$
\varsigma(\theta \breve{u}, \theta \breve{v}) \leq \varphi(\varsigma(\breve{u}, \breve{v})) \varsigma(\breve{u}, \breve{v}), \breve{u}, \breve{v} \in C(J) \text { with }(\breve{u}, \breve{v}) \in \Re \text {. }
$$

Thus, all assumptions of Theorem 17 are satisfied, so $\theta$ has a fixed point. Finally, in view of the proof of Theorem $19, \theta$ has a unique fixed point, which is indeed a unique solution of the problem (30)

\section{Conclusion}

In this work, we have proved new relational and graphical variants of fixed point results and validated all the assertions with the help of nontrivial examples. We have also provided a view to connect the theory of fixed point results equipped with a binary relation with that of graph related metrical fixed point theory. Further, inspired by the fact that boundary value problems appear in various branches of science and engineering, we resolve them to verify the genuineness and utility of the established conclusions.

\section{Data Availability}

The data used to support the findings of this study are available from the corresponding author upon request.

\section{Conflicts of Interest}

No competing interests are associated with the article.

\section{Authors' Contributions}

All authors done the equal contributions to the article.

\section{References}

[1] Y. I. Alber and S. Guerre-Delabriere, "Principles of weakly contractive maps in Hilbert spaces, new results in operator theory," in New Results in Operator Theory and Its Applications, I. Gohberg and Y. Lyubich, Eds., vol. 98 of Operator 
Theory: Advances and Applications, , pp. 7-22, Birkhauser Verlag, Basel, 1997.

[2] B. E. Rhoades, "Some theorems on weakly contractive maps," Nonlinear Analysis, vol. 47, no. 4, pp. 2683-2693, 2001.

[3] D. W. Boyd and J. S. W. Wong, "On nonlinear contractions," Proceedings of the American Mathematical Society, vol. 20, no. 2, pp. 458-464, 1969.

[4] M. Geraghty, "On contractive mappings," Proceedings of American Mathematical Society, vol. 40, no. 2, pp. 604-608, 1973.

[5] S. Reich, "Some fixed point problems," Atti della Accademia Nazionale dei Lincei, vol. 57, no. 3-4, pp. 194-198, 1974.

[6] P. N. Dutta and B. S. Choudhury, "A generalisation of contraction principle in metric spaces," Fixed Point Theory and Applications, vol. 2008, no. 1, Article ID 406368, 2008.

[7] A. Amini-Harandi and H. Emami, "A fixed point theorem for contraction type maps in partially ordered metric spaces and application to ordinary differential equations," Nonlinear Analysis, vol. 72, no. 5, pp. 2238-2242, 2010.

[8] S. Banach, "Sur les opérations dans les ensembles abstraits et leur application aux équations intégrales," Fundamenta Mathematicae, vol. 3, pp. 133-181, 1922.

[9] M. Turinici, "Fixed points for monotone iteratively local contractions," Demonstratio Mathematica, vol. 19, no. 1, pp. 171$180,1986$.

[10] A. C. M. Ran and M. C. B. Reurings, "A fixed point theorem in partially ordered sets and some applications to matrix equations," Proceedings of the American Mathematical Society, vol. 132, no. 5, pp. 1435-1443, 2004.

[11] J. J. Nieto and R. Rodríguez-López, "Contractive mapping theorems in partially ordered sets and applications to ordinary differential equations," Order, vol. 22, no. 3, pp. 223-239, 2005.

[12] F. Sabetghadam and H. P. Masiha, "Fixed point results for multi-valued operators in quasi-ordered metric spaces," Applied Mathematics Letters, vol. 25, no. 11, pp. 1856-1861, 2012.

[13] F. Sabetghadam, H. P. Masiha, and H. Aydi, "New results for set-valued mappings on ordered metric spaces," Journal of Function Spaces, vol. 2020, Article ID 5393521, 5 pages, 2020.

[14] J. Harjani and K. Sadarangani, "Fixed point theorems for weakly contractive mappings in partially ordered sets," Nonlinear Analysis, vol. 71, pp. 3403-3410, 2009.

[15] B. Samet and M. Turinici, "Fixed point theorems on a metric space endowed with an arbitrary binary relation and applications," Communications in Mathematical Analysis, vol. 13, no. 2, pp. 82-97, 2012.

[16] A. Alam and M. Imdad, "Nonlinear contractions in metric spaces under locally T-transitive binary relations," Fixed Point Theory, vol. 19, no. 1, pp. 13-24, 2018.

[17] A. Alam and M. Imdad, "Relation-theoretic contraction principle," Journal of Fixed Point Theory and Applications, vol. 17, no. 4, pp. 693-702, 2015.

[18] G. Prasad, "Fixed points of Kannan contractive mappings in relational metric spaces," Journal of Analysis, vol. 29, no. 3, pp. 669-684, 2021.

[19] G. Prasad, "Fixed point theorems via w-distance in relational metric spaces with an application," Filomat, vol. 34, no. 6, pp. 1889-1898, 2020.

[20] J. Jachymski, "The contraction principle for mappings on a metric space with a graph," Proceedings of American Mathematical Society, vol. 136, no. 4, pp. 1359-1373, 2008.
[21] M. Abbas, T. Nazir, T. A. Lampert, and S. Radenović, "Common fixed points of set-valued F-contraction mappings on domain of sets endowed with directed graph," Computational and Applied Mathematics, vol. 36, no. 4, pp. 1607-1622, 2017.

[22] N. Hussain, M. Arshad, A. Shoaib, and Fahimuddin, "Common fixed point results for $\alpha-\psi$-contractions on a metric space endowed with graph," Journal of inequalities and applications, vol. 2014, no. 1, 2014.

[23] O. Tripak, "Common fixed points of G-nonexpansive mappings on Banach spaces with a graph," Fixed Point Theory and Applications, vol. 2016, no. 1, Article ID 87, 2016.

[24] S. Lipschutz, Schaum's Outlines of Theory and Problems of Set Theory and Related Topics, McGraw-Hill, New York, 1964.

[25] B. Kolman, R. C. Busby, and S. Ross, Discrete Mathematical Structures, PHI Pvt. Ltd., New Delhi, Third Edition edition, 2000.

[26] M. Turinici, "Ran and Reuring's theorems in ordered metric spaces," The Journal of the Indian Mathematical Society, vol. 78, pp. 207-214, 2011.

[27] M. Turinici, "Contractive operators in relational metric spaces," in Handbook of Functional Equations, vol. 95, pp. 419-458, Springer, 2014. 